\title{
I: ÜBERLIEFERUNG
}

Im Zusammenhang einer möglichst vollständigen Sammlung ${ }^{1}$ der hochdeutschen Übersetzungen der Perikope Mt 13,44-53 in mittelalterlichen Handschriften konnten unter dem Titel 'Bremer Evangelistar' fünf Kodizes ermittelt werden². Eine Überprüfung am gesamten Text hat ergeben, daß die Berliner Handschrift - Staatsbibliothek Preußischer Kulturbesitz, Ms. germ. $4^{\circ} 2025$ - dieser Übersetzung trotz teilweiser Übereinstimmungen doch nicht zuzuordnen ist. Neu ist die Zuordnung der Frankfurter Handschrift (Sigle: Fr) - Stadt- und Universitätsbibliothek, Ms. germ. qu. 55 -, die meines Wissens mit diesem 'Übersetzungszweig' noch nicht in Verbindung gebracht worden ist. Nach MÄRTA ÁsDAHL HoLMBERG ist sie sogar bis Ende der 60er Jahre der "bisherigen Bibelforschung überhaupt nicht bekannt" ${ }^{3}$ gewesen. Der Bremer Kodex (Sigle: Br) - Staats- und Universitätsbibliothek, msa 0024 ist von WILLY LUDTKE im Rahmen seiner Untersuchungen 'Evangelientexte, besonders aus Harmonien'4 eingehend untersucht worden, und auf ihn stützt sich die Wahl des Titels, die wegen des neuen Textzeugen nicht geändert werden soll. Die beiden Nürnberger Handschriften (Siglen: N1 bzw. N2) - Stadtbibliothek, Cod. Cent. V, App.30 sowie Cod. Cent. VIII,2 - erwähnt LƠDTKE ${ }^{5}$ in diesem Zusammenhang zwar neben anderen auch, ohne allerdings den gesamten Text überprüft zu haben. Indem für ausgewählte Stellen Übereinstimmungen oder auch nur ungefähre Entsprechungen mit anderen Handschriften konstatiert werden, ist keine Klarheit zu gewinnen. Dies gilt insgesamt für die Tabellen in den Bänden 'Bibel und deutsche Kultur', ungeachtet ihres Wertes für erste Zugriffe in dem auch heute

1 Vgl. JOCHEN SPLETT (Hrsg.), das hymelreich ist gleich einem verporgen schatz in einem acker ..., Göppingen 1987.

2 SPLeTT (wie Anm. 1), S. 29*f.; ders., in: Vestigia Bibliae 9/10 (1991), S. $56 f$.

3 Exzipierend-einschränkende Ausdrucksweisen, Uppsala 1967, S. $173 f$.

4 In: BdK 11 (1941), S. 320, und die Fortsetzung in seiner Monographie gleichen Titels, Hamburg 1965.

5 Evangelientexte, besonders aus Harmonien, Hamburg 1965, S. $16 \mathrm{ff}$.

6 Vgl. die Auflistung der Bände dieser sehr uneinheitlichen Veröffentlichungsreihe bei SPLETT (wie Anm. 1), S. 48*f.; hier vor allem Band 5 (1935). 
noch unüberschaubaren Gebiet vorlutherischer Bibelübersetzung. Dies zeigt sich etwa, wenn BirgitT WeImanN ${ }^{7}$ auf HaNS Vollmers Tabellen hinweist, die u. a. Verbindungen zu den Olmützer Perikopen (Cgm 58, Cgm 66 und Berlin Ms. germ. $\left.4^{\circ} 1335\right)^{8}$ hervorheben, aber die Bremer und die beiden Nürnberger Handschriften nicht heranziehen. Ungeachtet der Tatsache, daß möglicherweise neue Textzeugen auftauchen, ist nur durch die Edition vor allem vollständiger Perikopenbücher Schritt für Schritt ein sicherer Boden zu gewinnen. Die Ausgabe 'Das Leben Jhesu' durch CHRISTOPH GERHARDT ${ }^{9}$ war ein solcher Schritt, dem aber bisher offensichtlich keine weiteren von anderer Seite gefolgt $\operatorname{sind}^{10}$. Lediglich $\cdot \mathbf{A b}$ drucke einzelner Handschriften ${ }^{11}$ oder Teileditionen ${ }^{12}$, so nützlich sie auch sein mögen, helfen kaum weiter, die disparate Überlieferung dieser Textsorte aufzuhellen.

\section{BESCHREIBUNG DER HANDSCHRIFTEN ${ }^{13}$}

\subsection{Frankfurt / Main, Stadt- und Universitätsbibliothek, Ms. germ. qu. 55 (olim: Ms. II, 29) ${ }^{14}$}

Die Papierhandschrift im Format 29,5 cm x 19,5cm aus der zweiten Hälfte des 14. Jahrhunderts umfaßt 94 Blätter mit je einem modernen Vorsatz- und Nachstoßblatt. Den Aufbau des Kodex, dessen Blattränder besonders am Anfang teilweise beschädigt sind, veranschaulicht die folgende Lagenformel: $1+(\text { III }-1)^{6}+\mathrm{IV}^{14}+(\mathrm{IV}-1)^{21}+\mathrm{IV}^{29}+\mathrm{II}^{33}+2 \mathrm{IV}^{49}+(\mathrm{IV}$ $-1)^{56}+2 \mathrm{IV}^{n}+\mathrm{II}^{88}+2 \mathrm{IV}^{94}$. Er ist am rechten oberen Rand von moderner Hand mit Bleistift durchfoliiert. Die alte Lagenzăhlung mit römischen Ziffern findet sich jeweils auf

7 Die mittelalterlichen Handschriften der Gruppe Manuscripta germanica (= Kataloge der Stadt- und Universitätsbibliothek Frankfurt am Main. Bd. 5: Die Handschriften der Stadt- und Universitätsbibliothek Frankfurt am Main IV), Frankfurt / Main 1980, S.64.

8 Vgl. SpletT (wie Anm. 1), S.19*-23*.

9 Leiden 1970. Dazu seine Untersuchungen unter demselben Titel, München 1969.

10 Vgl. Hemmo Reinitzer / Olaf Schwencke, 'Plenarien'. In: VL, 2. Auflage, Bd. 7, 1989, Sp.737ff.

11 So etwa die Edition des Evangelistars der Berliner Handschrift Ms. germ. $4^{\circ} 533$ durch GÖNTER FEUDEL, der allerdings damit andere Ziele verfolgt hat.

12 Vgl. etwa HaNs JeSKE, Der Kodex Trier 810/1338, Uppsala 1974.

13 Die Handschriftenbeschreibungen beruhen auf der jeweils angeführten kodikologischen Literatur, die anhand eines Mikrofilms und durch Autopsie überprüft, ergänzt und berichtigt worden sind.

14 B. Wemann (wie Anm. 7), S. 62-64. 
dem ersten Lagenblatt links unten, und zwar beginnend mit ii auf den Blättern 7, 15, 22, 30, 34, 42, 50, 57, 65 und 73. Die Zählung der Doppelblätter mit arabischen Ziffern innerhalb der einzelnen Lagen - jeweils auf der Rectoseite rechts oben, und zwar jeweils ab zweitem Doppelblatt - stammt von der Hand des Schreibers. Die beiden letzten Lagen sind bereits jeweils ab dem ersten Doppelblatt gezăhlt, und zwar mit römischen Ziffern, die allerdings durch Beschneiden der Blattränder zum Teil unvollständig, zum Teil weggefallen sind. Die Wasserzeichen zeigen zwei Kreise mit einer durchgehenden, geraden Linie, die in einem Kreuz endet, und ähneln dem bei Briquet 3190 (a.1349-1351) abgebildeten. Darüber hinaus finden sich Reste von zwei oder drei Wasserzeichen.

Der Schriftraum der zweispaltig geschriebenen Handschrift beträgt $22,7 \mathrm{~cm}$ bis $24 \mathrm{~cm}$ $x 15,5 \mathrm{~cm}$ bis $14 \mathrm{~cm}$. Die Kolumnen umfassen fast durchgehend 28 Zeilen mit folgenden Abweichungen:
29 Zeilen: 5r, 14v
33 Zeilen: 7rv, 8r, 9v, 12r, 13v, 14r
30 Zeilen: 4v, 6
34 Zeilen: 2rv, 3r, 8v
31 Zeilen: 4r, 5v
35 Zeilen: 10rv, 11rv.
32 Zeilen: 3v, 9v, 12v, 13r, 77r

Die Zirkellöcher auf dem Außenrand der Blätter sind zumeist noch deutlich zu erkennen. Die Handschrift ist von zwei eindeutig unterscheidbaren, aber stilähnlichen Händen mit sehr dunkler brauner Tinte geschrieben. Der zweiten Hand sind fol 49rv und fol 75v bis Textende auf fol 93v zuzuordnen. Es handelt sich um eine Textualis mittleren Niveaus - Kasten- $a$ und doppelbäuchiges $a$, 'offizielles' Schluß-s, vereinzelt Zierstriche. In der ersten Zeile jeder Spalte sind vielfach einzelne Oberlängen nach Art der Urkundenschrift verlängert und mit rotem Schnörkelwerk ausgeschmückt.

Die Überschriften sind rot, die roten Lombarden am Beginn der einzelnen Perikopen mehr-, zumeist zweizeilig. Bei lateinischem Initium wird das Einsetzen der deutschen Übersetzung jeweils durch eine einzeilige rote Lombarde hervorgehoben. Besonders ausgeschmückt sind die Lombarden bei Beginn des Textes (2ra), zur Osterperikope (48ra), zur Perikope am Montag nach dem ersten Sonntag nach Ostern (52ra), zur Pfingstperikope (58rb), zur Perikope an Trinitatis (61ra), zu der Perikope am achten Sonntag (67va), zur Freitagsperikope in der Woche nach dem fünfzehnten Sonntag (74rb) und zur Perikope am sechzehnten Sonntag jeweils nach Pfingsten (75ra), bei Beginn des Sanktorale (79ra), zu den Perikopen an den Marienfesten Himmelfahrt (82vb) und Geburt (83va), zur Perikope an Allerheiligen (91vb) und zu der am Fest einer heiligen Jungfrau (92va). Mit einem senkrechten roten Strich sind die Anfangsbuchstaben der Wörter, mit denen ein Satz bzw. ein Satzteil beginnt, vom Rubrikator durchgehend, aber unregelmäßig und nicht immer konsequent gekennzeichnet. 
Die in neuerer Zeit restaurierte Handschrift - Risse in den Blättern 21, 29, 64 und 84, die bis in den Text hineinreichen, sind mit durchsichtigen Papierstreifen überklebt - ist in einen modernen Halbpergamentband gebunden. Der Kodex stammt aus der Bibliothek des Frankfurter Ratsherren und Schöffen Johann(es) Maximilian(us) zum Jungen (1596-1649), die der Frankfurter Rat Ende 1689 oder Anfang 1690 von dessen Erben erworben hat ${ }^{15}$.

\subsection{Bremen, Staats- und Universitätsbibliothek, msa $0024^{16}$}

Die Papierhandschrift im Format $29 \mathrm{~cm} \times 19,5 \mathrm{~cm}$ aus Anfang des 15. Jahrhunderts (vor a.1419) umfaßt 133 Blätter. Den Aufbau veranschaulicht die folgende Lagenformel: $(I+1)^{2}$ $+(\mathrm{IV}+1)^{11}+(\mathrm{IV}+1)^{20}+2 \mathrm{IV}^{36}+(3+\mathrm{I})^{41}+\mathrm{II}^{45}+\mathrm{IV}^{53}+(\mathrm{IV}+\mathrm{I})^{63}+(\mathrm{I}+\mathrm{III}$ $+1)^{72}+\mathrm{IV}^{80}+(1+\mathrm{III})^{87}+(1+\mathrm{I})^{90}+(\mathrm{III}+\mathrm{I}+\mathrm{I}+2)^{102}+\mathrm{II}^{106}+(\mathrm{III}+1)^{113}+$ $\mathrm{III}^{119}+(1+\mathrm{V})^{130}+\mathrm{II}^{133}$. Das erste Blatt bzw. das letzte Blatt bilden die Innenspiegel. Das erste Doppelblatt (fol.12/20) besteht aus zwei ursprünglichen Einzelblättern, und fol.18 ist ein Einzelblatt. Das Doppelblatt (fol.62/63) ist mit einem Falz an fol.53 angeklebt. Das Doppelblatt (fol.64/65) ist durch einen Falz mit dem Einzelblatt (fol.72) verbunden. Das Einzelblatt (fol.89) ist eingeschoben. Die zwei Doppelblătter (fol.94/95 bzw. fol.96/97) und die nachfolgenden beiden Einzelblätter sind eingeschoben. Die folgende Tabelle gibt einen Überblick über die Foliierung mit arabischen Ziffern - jeweils am rechten oberen Rand -, die teilweise gestörte Foliierung des Evangelistars mit roten römischen Ziffern - jeweils etwa in der Mitte des oberen Randes -, die nur noch resthaft vorhandenen Lagenbezeichnungen - am oberen Rand über der zweiten Kolumne bzw. ab 67r oben über dem Interkolumnium - und die Verteilung der Schreiberhände:

15 Vgl. auch KURT OHLY / VERA SACK, Inkunabelkatalog der Stadt- und Universitätsbibliothek und anderen öffentlichen Sammlungen in Frankfurt am Main, Frankfurt / Main 1967, S. 698f.

16 W. LÖDTKE, Evangelientexte, besonders aus Harmonien, Hamburg 1965, S. 19-30. Für briefliche Auskünfte zur neuen Schreibkonvention der Bremer Handschriftensignaturen, zur genauen Lagenanordnung, zum Einband und zur Provenienz der vorliegenden Handschrift sowie für den Literaturhinweis auf den Katalog des Focke-Museums - Schätze der Stadt Bremen in der Universitätsbibliothek. Miniaturen und Handschriften des Mittelalters, Bremen 1975 - mit einer kurzen Handschriftenbeschreibung (Nr. 20) danke ich dem Leiter der Staats- und Universitātsbibliothek Bremen, Herm Priv.-Doz. Dr. Armin Hetzer. Die Angaben zu den Wasserzeichen, die erst im Rahmen der Restaurierung ermittelt werden konnten, verdanke ich Herrn Dr. Helmar HÃRTEL von der Herzog August Bibliothek Wolfenbüttel. 


Foliierungen Lagenbez. Hände Bemerkungen

\begin{tabular}{|c|c|c|c|c|}
\hline 1 & & & & $\begin{array}{l}\text { Ir unbeschrieben; } 1 \mathrm{v} \text { modernes Inhaltsver- } \\
\text { zeichnis }\end{array}$ \\
\hline $2-3$ & & & 7. Hand & unbeschrieben; 3v Kaufvermerk \\
\hline $4-6$ & & & 6. Hand & \\
\hline $7-9$ & & & & unbeschrieben \\
\hline $10-11$ & & & 5. Hand & 10r unbeschrieben \\
\hline $12-13$ & I-II & & 2. Hand & \\
\hline $14-18$ & III-VII & & 1. Hand & \\
\hline 19 & VIII & & 2./3. Hand & 3. Hand $19 \mathrm{vb}$ \\
\hline 20 & & & 3./5. Hand & 5. Hand ab 20ra, Z.15 \\
\hline $21-22$ & XV-XVI & & 1. Hand & \\
\hline 23 & XVII & 3 & 1. Hand & \\
\hline 24 & XVIII & 4 & 1. Hand & \\
\hline $25-28$ & XIX-XXII & & 1. Hand & \\
\hline 29 & & & 5. Hand & \\
\hline $30-36$ & XXIIII-XXX & & 1. Hand & \\
\hline $37-38$ & XXX-XXXI & & 1. Hand & \\
\hline 39 & XXXII & 3 & 1. Hand & \\
\hline 40 & XXXIIII & 4 & 1. Hand & \\
\hline 41 & XXXV & & 1. Hand & \\
\hline $42-45$ & & & 5. Hand & \\
\hline $46-47$ & XXXVIII-XXXIX & & 1. Hand & \\
\hline 48 & $\mathrm{XL}$ & 3 & 1. Hand & \\
\hline 49 & XLI & 4 & 1. Hand & \\
\hline $50-51$ & XLII-XLIII & & 1. Hand & \\
\hline 52 & XLIII & & 1. Hand & XLV getilgt (durchgestrichen) \\
\hline $53-54$ & XLIIII-XLV & & 1. Hand & \\
\hline 55 & XLVI & 2 & 1./3. Hand & $\begin{array}{l}\text { 3. Hand 55va, Z.1-10 (auf aufgeklebtem } \\
\text { Papierzettel) }\end{array}$ \\
\hline 56 & XLVII & 3 & 1. Hand & \\
\hline 57 & XILVIII & 4 & 1. Hand & \\
\hline 58 & XLIX & & 1. Hand & \\
\hline 59 & & & 1. Hand & \\
\hline
\end{tabular}


Lagenbez. Hände Bemerkungen

\begin{tabular}{|c|c|c|c|c|}
\hline 60 & L & & 1. Hand & \\
\hline $61-65$ & & & 5. Hand & \\
\hline 66 & & & 1. Hand & \\
\hline 67 & LII & 3 & 1. Hand & Lagenbez.: 2 Punkte, 1 Strich \\
\hline 68 & LIII & 4 & 1. Hand & Lagenbez.: 3 Punkte, 1 Strich \\
\hline $69-70$ & LIIII-LV & & 1. Hand & \\
\hline 71 & LVI & & 1. Hand & LXII getilgt (durchgestrichen) \\
\hline 72 & & & 5. Hand & \\
\hline 73 & LVIII & & 1. Hand & LXIIII getilgt (durchgestrichen) \\
\hline 74 & LIX & 2 & 1. Hand & $\begin{array}{l}\text { Lagenbez.: } 2 \text { Punkte; LXX getilgt (durch- } \\
\text { gestrichen) }\end{array}$ \\
\hline 75 & LX & 3 & 1. Hand & $\begin{array}{l}\text { Lagenbez.: } 3 \text { Striche; LXXI getilgt } \\
\text { (durchgestrichen) }\end{array}$ \\
\hline 76 & LXI & 4 & 1. Hand & $\begin{array}{l}\text { Lagenbez.: } 4 \text { Striche; LXXII getilgt } \\
\text { (durchgestrichen) }\end{array}$ \\
\hline 77 & LXII & & 1. Hand & LXXIII getilgt (durchgestrichen) \\
\hline 78 & LXIII & & 1. Hand & LXIX getilgt (durchgestrichen) \\
\hline 79 & LXIIII & & 1. Hand & LXX getilgt (durchgestrichen) \\
\hline $80-81$ & LXXI-LXXII & & 1. Hand & \\
\hline 82 & LXXIII & 2 & 1. Hand & Lagenbez.: 2 Punkte \\
\hline 83 & LXXIIII & 3 & 1. Hand & Lagenbez.: 3 Striche \\
\hline $84-87$ & LXXV-LXXVIII & & 1. Hand & \\
\hline $88-106$ & LXXIX-XCVII & & 2.15. Hand & 5. Hand 106v \\
\hline 107 & XCVIII & 2 & 1. Hand & Lagenbez.: 2 Striche \\
\hline 108 & $\mathrm{XCIX}$ & & 1. Hand & \\
\hline 109 & $\mathrm{C}$ & 3 & 1. Hand & Lagenbez.: 3 Striche \\
\hline $110-114$ & $\mathrm{CI}-\mathrm{CV}$ & & 1. Hand & \\
\hline 115 & CVI & 2 & 1. Hand & Lagenbez.: 2 Striche \\
\hline $116-117$ & CVII-CVIII & & 1. Hand & \\
\hline 118 & CIX & & 2./5. Hand & 5. Hand $118 v$ \\
\hline $119-121$ & CX-CXII & & 5. Hand & \\
\hline 122 & & & 5. Hand & \\
\hline $123-133$ & & & & unbeschrieben \\
\hline
\end{tabular}


Eine vierte Hand hat außer Verbesserungen im Text auch Änderungen auf Papierzettel geschrieben und diese über den zu verbessernden Text geklebt, und zwar auf den Seiten: 13rb (Z.19-26), 18ra (Z.17-27), 21vb (Z.10-11), 38ra (Z.4), 39ra (Z.24), 40vb (Z.12), 41va (Z.4, Z.10), 47vb (Z.25), 48ra (Z.1-6), 55ra (Z.14-16), 55vb (Z.15), 56ra (Z.1), 66ra (Z.14, Z.17), 66rb (Z.1), 66va (Z.3), 66vb (Z.23-24), 67rb (Z.17-20), 69ra (Z.5, Z.14-22), 70rb (Z.23-27), 71rb (Z.11), 71va (Z.15), 71vb (Z.12-20), 73va (Z.17-20), 74rb (Z.15), 74va (Z.11-16), 76va (Z.14-17), 81ra (Z.8-11, Z.17-18), 81va (Z.21), 86ra (Z.10-16), 91rb (Z.22-24), 91va (Z.1-4), 94rb (Z.10), 94vb (Z.9-12), 95rb (Z.13 ?), 99va (Z.9), 107vb (Z.8-23), 111ra (Z.6-13), 114ra (Z.1-4). Zudem stammen die Nachträge auf dem Rand von Seite 50r und 54r von ihr. Von der fünften Hand geschriebene Textänderungen auf aufgeklebten Papierzetteln finden sich auf den Seiten 55vb (Z.16-22) und 66vb (Z.15-17). Um einzelne Buchstaben, Wörter oder Zeilen des verworfenen Textes zu löschen, sind unbeschriebene bzw. mit Strichen und Punkten versehene Papierstūcke aufgeklebt: 21ra (Z.1-3), 25va (Z.19), 30va (Z.20), 50ra (Z.20), 74rb (Z.14), 107ra (Z.1).

Auf Anfrage bei der Herzog August Bibliothek in Wolfenbüttel, wo die Handschrift zur Zeit restauriert wird, lassen sich an Wasserzeichen ein Ochsenkopf (Piccard VII 609: a.1419-1424) und ein Schlüssel (Piccard V 43: a.1417) verifizieren. Nicht zuzuordnen sind die Wasserzeichen Kreis, Krone, Wappen und ein weiterer Ochsenkopf.

Der Schriftraum dieser zweispaltig geschriebenen Handschrift beträgt etwa $19 \mathrm{~cm}$ bis $20,5 \mathrm{~cm} \times 13 \mathrm{~cm}$ bis $15 \mathrm{~cm}$. Die Zeilenzahl der Kolumnen des Perikopenregisters (4ra-6va) ist unterschiedlich und schwankt zwischen 39 und 46 Zeilen. Sonst umfaßt eine Kolumne in der Regel 26 Zeilen, die auf einer Seite nicht immer auf gleicher Höhe stehen. Folgende Kolumnen bzw. Seiten haben eine abweichende Zeilenzahl, was zumindest teilweise auf die aufgeklebten Textverbesserungen zurückzuführen ist:

20 Zeilen: 11va, 19rb, 19vb

21 Zeilen: 13ra, 19ra, 20ra, 64rb-65rb, 95ra-95va, 96ra, 100vb, 105vb, 106rb, 118ra

22 Zeilen: 44ra, 64ra, 92rb, 94rb, 96rb-97ra, 97va, 102vb-103ra, 105va, 106ra

23 Zeilen: 19va, 44rb-44vb, 63ra, 65va, 72r, 89r, 90va-91ra, 92ra, 93ra-94ra, 94va, 97rb, 97vb-98rb, $99 \mathrm{ra}, 99 \mathrm{va}-100 \mathrm{ra}, 100 \mathrm{vb}-101 \mathrm{rb}, 103 \mathrm{rb}-103 \mathrm{vb}, 104 \mathrm{rb}, 107 \mathrm{vb}-108 \mathrm{ra}, 119 \mathrm{vb}$

24 Zeilen: 10va, 13v, 43v, 45r, 65vb, 88ra-88va, 89vb-90ra, 91rb-91vb, 92vb, 94vb, 98va-99rb, 100va, $102 \mathrm{ra}, 104 \mathrm{ra}, 104 \mathrm{va}-105 \mathrm{rb}, 107 \mathrm{va}, 108 \mathrm{rb}, 109 \mathrm{ra}-110 \mathrm{vb}, 111 \mathrm{va}-112 \mathrm{rb}, 113 \mathrm{ra}-113 \mathrm{vb}, 115 \mathrm{v}$, $116 \mathrm{va}, 117 \mathrm{rb}, 121 \mathrm{rb}$

25 Zeilen: 11ra, 12ra-12vb, 21ra, 38r, 42vb, 43rb, 46ra-47vb, 48rb-53va, 61ra, 88vb, 89va, 90rb, 92va, $100 \mathrm{rb}, 101 \mathrm{v}, 102 \mathrm{rb}-102 \mathrm{va}, 107 \mathrm{r}, 108 \mathrm{v}, 111 \mathrm{r}, 112 \mathrm{v}, 114 \mathrm{rb}-115 \mathrm{rb}, 116 \mathrm{r}, 117 \mathrm{v}, 118 \mathrm{va}-119 \mathrm{ra}$, $119 \mathrm{va}, 120 \mathrm{r}, 120 \mathrm{vb}, 121 \mathrm{va}$

27 Zeilen: 18ra, 55vb, 62ra, 69ra, 70rb, 74va, 81ra

28 Zeilen: 71vb, 72v, 86ra

29 Zeilen: 48ra, 54rb, 61v 
30 Zeilen: 20rb, 29vb

31 Zeilen: 10vb.

Seite 20v ist bis auf 2 Zeilen in der ersten Kolumne (Perikopenschluß) unbeschrieben. Auf Seite 45v endet die 5. Hand (erste Kolumne, Zeile 11) und der Text setzt - geschrieben von der 1. Hand - auf Seite 46ra wieder ein. Die Kolumne 106vb endet mit der 13. Zeile, dem Ende des Zusatzes. Der Text in Kolumne 118ra bricht mitten im Satz ab, die Kolumne 118rb ist unbeschrieben und mit Zeile 8 endet auf Seite 122ra der Text der Handschrift. Die schadhaften Ränder sind vielfach mit Bütten bzw. Ziegenleder überklebt. Diese Randverstärkungen sind wohl bereits mittelalterlich, da teilweise die rote Foliierung und Textverbesserungen auf ihr stehen. Die Zirkellöcher auf dem Außenrand der Blätter sind nur noch vereinzelt erhalten.

Es lassen sich 7 Hände unterscheiden. Die erste ist eine nicht sehr sorgfältig geschriebene Textualis, die zweite eine etwas größere und dickere, nicht so eng geschriebene und regelmäßigere Textualis. Die dritte Hand - eine schlankere Textualis - schreibt im Vergleich zu den beiden Haupthänden nur wenige Zeilen (19vb - 20ra, Z.14 und 55va, Z.1-10). Die Korrekturhand (vgl. oben) hat einen ähnlichen Duktus wie die dritte Hand. Die fünfte Hand ist eine Buchkursive, ebenso die sechste, die das Perikopenregister und die Foliierung mit roten römischen Ziffern geschrieben hat. Von der siebten Hand, einer der fünften Hand āhnlichen Kursivschrift, stammt der Kaufvermerk (3v): Diz buch wart kauft vm(m)e xiiij $\beta$ [Schilling] nach S(anc)te paulus dag als er bekart wart [25. Januar] jm xiiif vnd xix jar [a.1419]. Ob der Zusatz auf Seite 120rb einer achten Hand ${ }^{17}$ zuzuordnen ist, oder ob hier die fünfte Hand mit einer spitzeren Feder geschrieben hat, ist fraglich.

Die Überschriften im Evangelistar sind rot, die Lombarden am Beginn der einzelnen Perikopen mehr-, zumeist zweizeilig. Bei lateinischem Initium wird die deutsche Übersetzung fast durchgehend jeweils durch eine einzeilige Lombarde hervorgehoben.

Der Einband besteht aus Kleisterpapier mit Rücken und Ecken in Ziegenpergament. Er hat Doppelbundheftung auf Kordel, der Rücken ist mit lat. Fragmenten in karolingischer Minuskel hinterklebt. Über die Provenienz ist nichts überliefert.

Außer dem Evangelistar (12ra - 122ra) enthält die Handschrift ${ }^{18}$

4ra - 6va, Z.8:

zweispaltiges Perikopenregister

10v:

Lucidarius (S.49, Z.13 - S.50, Z.8)

11ra:

Epistel zum 1. Adventssonntag (Römer 13,11-14)

11rb:

Epistel zur ersten Messe am 25. Dezember (Titus 2,11-14)

17 So W. LÖDTKE (wie Anm. 16), S. 20.

18 Außer dem Perikopenregister, der ersten Epistel und dem Predigtfragment abgedruckt bei W. LODTKE (wie Anm. 15), S. 26ff. Vgl. den Abdruck aller Zusätze hier im Anhang, S. 365ff. 
11va:

63vb, Z.13-18:

63vb, Z.20-26:

65rb, Z.5-16:

65rb, Z.17-21:

65vb, Z.15-24:

106va, Z.1-10:
Bruchstūck einer Predigt

Jz sprichet der prophete in dem selter ... mit dragheit - Nicht im Psalter; Anklänge an Römer 1,29 und Ecclesiasticus 1,40. Psalm 41,2 (42,2)

Jz sprichet sanctus Jeronimus ... gent zu gerechte

Sancte gregorius ... zu irgrundene

Der groz meister bischoff albrecht ... tut ubere dem altere

Lucidarius (S.41, Z.4-7)

106va, Z.11 - 106vb, Z.13: Lucidarius (S.35, Z.6-21 mit geringfügigen Auslassungen und Umformungen)

120rb, Z.5-25:

120vb, Z.14-25:

sancte bernhart sprichet ... davon niemant gesagen

Psalm 41,3f. (42,3f.)

121rb, Z.15 - 121va, Z.6: Von drin sachen ... der heilgen driualdekeit.

\subsection{Nürnberg, Stadtbibliothek,}

Cod. Cent. V, App. 30 (olim: Cod. D XII) ${ }^{19}$

Die Papierhandschrift im Format $28,2 \mathrm{~cm} \times 20 \mathrm{~cm}$ aus dem ersten Viertel des 15. Jahrhunderts umfaßt 96 Blätter. Den Aufbau des Kodex veranschaulicht die folgende Lagenformel: $4 \mathrm{~V}^{40}+\mathrm{VI}^{52}+3 \mathrm{~V}^{82}+\mathrm{IV}^{90}+\mathrm{II}^{96}$. Er ist am rechten oberen Rand von alter Hand mit roten arabischen Ziffern durchfoliiert. Das Sanktorale hat zusätzlich von der gleichen Hand eine Foliierung in dunkelbraunen arabischen Ziffern jeweils auf der Versoseite links oben. Sie beginnt auf der Seite 82v und endet auf Seite 94v; auf Seite 91v fehlt die Folioangabe 10. Die drei bis vier Wörter umfassenden Reklamanten stehen jeweils am Ende der Lagen, sind aber größtenteils bis auf einzelne noch erkennbare Oberlängen abgeschnitten. Die Wasserzeichen zeigen einen Ochsenkopf mit Andreaskreuz und ähneln dem bei Briquet 14613 (a.1373) abgebildeten.

Der Schriftraum der zweispaltig geschriebenen Handschrift beträgt $17 \mathrm{~cm}$ bis $18 \mathrm{~cm}$ x $12 \mathrm{~cm}$ bis $12,5 \mathrm{~cm}$. Die Höhen der Kolumnen auf einer Seite sind mitunter verschieden, die Zeilen stehen nicht immer auf gleicher Höhe und differieren auf einer Seite häufig um eine, manchmal um zwei Zeilen. Die Zeilenzahl schwankt zwischen 24 und 27 mit folgenden Abweichungen: 23 Zeilen: 32rb, 35rb; 28 Zeilen: 2vb, 3vb, 15va, 90va.

19 KARIN SCHNEIDER / HENnZ ZIRNBAUER, Die deutschen mittelalterlichen Handschriften (= Die Handschriften der Stadtbibliothek Nürnberg I), Wiesbaden 1965, S. 71 . 
Der Kodex ist von nur einer Hand mit dunkelbrauner Tinte in Buchkursive geschrieben. Eine Hand des späten 15. Jahrhunderts hat in einer flüchtigen Kursive (Notula) auf den Rändern (4r, 7v, 10r, 13rv, 25v, 29r, 42v, 43v-45r, 88r, 89v-90v, 92v-94v) und am Ende (95vb, Z.16-24 und einspaltig 96r) Ergänzungen eingetragen. Auf dem unbeschriebenen Blatt 1 steht oben auf der Rektoseite von moderner Hand in deutscher Schrift Cent. V. Append. Nr. 30. / 96 Blatter enthaltend. Die alte Bibliothekssignatur D.XII• ist dagegen von einer Hand des 15. Jahrhunderts in roter Tinte auf dem oberen Rand von Seite $2 \mathrm{r}$ vermerkt.

Die Überschriften sind rot, die roten Lombarden am Beginn der einzelnen Perikopen mehr-, zumeist zweizeilig. Bei lateinischem Initium wird nur vereinzelt der Anfang der deutschen Übersetzung durch eine einzeilige Lombarde kenntlich gemacht. Mit einem senkrechten roten Strich sind die Anfangsbuchstaben der Wörter, mit denen ein Satz bzw. ein Satzteil beginnt, vom Rubrikator durchgehend, aber unregelmäßig und nicht immer konsequent gekennzeichnet. Das lateinische Initium auf Seite $2 \mathrm{r}$ ebenso wie die vorangehenden beiden Überschriften sind durch Auszeichnungsschrift, eine sorgfältig geschriebene Textualis, hervorgehoben. Die zugehörige Initiale $\mathrm{E}$ ist $6,8 \mathrm{~cm} \times 7,2 \mathrm{~cm}$ groß, rot und blau geviertelt mit ausgespartem gelb-grün koloriertem Lindenblattmuster und weißen Punkten. Die Federfleuronée-Füllung, -Umrandung und -Randleiste weisen gelbgrundige Lindenblattmedaillons auf. Nach H. ZIRNBAUER ${ }^{20}$ stammt diese nürnbergische, unter oberrheinischem Einfluß (?) stehende Ausschmückung nicht aus dem Katharinenkloster.

Der alte Koperteinband besteht aus einem dünnen Pergamentumschlag, dessen rechte untere Ecke am Vorderdeckel angesetzt ist. Der Rücken ist durch zwei Hornplättchen verstärkt. Auf dem Vorderdeckel befinden sich zwei braune Lederknöpfe zum Schließen, die Umschlagklappe am Rückendeckel fehlt. Im Vorderdeckel ist innen ein Pergamentblatt aufgeklebt, auf dem von der Hand der Schwester Kunigund Niklasin ${ }^{21}$ der Handschrifteninhalt vermerkt ist: It(em) an dem puch ste $(n) t$ / Die ew(an)gelio vo(n) d(er) czeit / Darnach stet ei(n) teil ew(an)/gelio vo(n) den heiligen. Darüber steht ein alter Bibliotheksvermerk Daz puch gehort in daz Clost(er) zu sant / kathr in nur p(re)dig(er) ord(en). Auf Seite 96v unten ist dieser Vermerk wiederholt: Das puch gehort jn das Closter zv sant kathrein p(re)diger orden / jn nvrmbergk. Oben auf derselben Seite ist als Federprobe ein kursives Alphabet von $a$ bis $o$ eingetragen. Auf dem Vorderdeckel steht oben ein Besitzvermerk des 15. Jahrhun-

20 K. SChNeIDER / H. ZIRNBAUer (wie Anm. 19), S. 72. Er verweist auf das Kloster Schönensteinbach (El$\mathrm{saß)}$, von dem her a.1428 das Katharinenkloster reformiert worden ist.

21 Schriftproben bei K. SCHNEIDER / H. ZIRNBaUER (wie Anm. 19), S. XVII, und auf den Tafelabbildungen Nr. 1-7. 
derts: Swest(er) margaretha im hoff. Die Handschrift ist von Margareta Imhoff $(\dagger 1468)$ ins Nürnberger Katharinenkloster der Dominikanerinnen eingebracht worden ${ }^{2}$.

\subsection{Nürnberg, Stadtbibliothek, Cod. Cent. VIII,2 (olim: Cod. D XIV) ${ }^{23}$}

Die Papierhandschrift im Format $21,5 \mathrm{~cm} \times 14,5 \mathrm{~cm}$ aus dem ersten Viertel des 15. Jahrhunderts umfaßt 125 Blätter. Den Aufbau des Kodex veranschaulicht die folgende Lagenformel: $10 \mathrm{VI}^{120}+(\mathrm{IV}-3)^{125}$. Er ist am rechten oberen Rand von moderner Hand mit Bleistift durchfoliiert. Die alte Lagenbezeichnung durch Kardinal- und Ordinalzahlen in arabischen Ziffern $\left(1,2,33^{9}, 4^{9}, 5,6,7^{9}\right.$ bis $\left.10^{9}\right)$ steht unten rechts am Rand jeweils auf der ersten Rectoseite, und zwar nur bei den zehn Sexternionen. Außerdem wird der Lagenwechsel durch ein bis drei Wörter umfassende Reklamanten jeweils am Lagenende angezeigt, aber nur auf den Seiten 12v, 24v, 48v, 108v und 120v. Da die Reklamanten in reichlichem Abstand von der Blattunterkante stehen, dürften die fehlenden auf den Seiten 36v, 60v, 72v, $84 v$ und 96v wohl kaum durch Beschneiden weggefallen sein. Die Wasserzeichen zeigen einen Ochsenkopf mit Andreaskreuz - ähnlich Briquet 14614 (a.1374), 14632 (a.1389) oder 14653 (a.1461) -, eine Blume - ähnlich Briquet 6486 (a.1392) -, eine Glocke - ähnlich Briquet 3967-3982 (a.1392 bis a.1423) - und einen Bogen - ähnlich Briquet 788-799 (a.1380 bis a.1406).

Der Schriftraum der einspaltig geschriebenen Handschrift beträgt $16 \mathrm{~cm}$ bis $16,6 \mathrm{~cm}$ $x 9,5 \mathrm{~cm}$ bis $10 \mathrm{~cm}$ und ist durch zwei senkrechte und zwei waagerechte Striche, die über die ganze Seite reichen, markiert. Vielfach wird die rechte Zeilenbegrenzung nicht eingehalten. Die Zeilenzahl schwankt zwischen 25 und 28 mit folgenden Abweichungen:

20 Zeilen: 59v, 63v

21 Zeilen: 57v, 61v, 64r, 65rv, 67r

22 Zeilen: 53v, 58rv, 59r, 60v, 61r, 62rv, 64v, 66rv

22 Vgl. Mittelalterliche Bibliothekskataloge Deutschlands und der Schweiz. Bd. III,3: Bistum Bamberg, bearbeitet von PAUL RUF, München 1939, S. 603: "D XII. Item ein puch in eim conpert; das helt in im die ewangelio. Die II vor geschriben plicher procht swester Margret Ymhoff zum closter." Vgl. auch K. SCHNEIDER / H. ZRRNBAUER (wie Anm. 19), S. XIV u. S. 47, sowie die dort angeführte Literatur. Keine weiterführenden Angaben bei SIGRID KRÄMER, Handschriftenerbe des deutschen Mittelalters, Teil 2, München 1989, S. 619.

23 K. Schneider / H. Zirnbauer (wie Anm. 19), S. 412 f. 
23 Zeilen: 26v, 52rv, 54rv, 55v, 56rv, 57r, 60r, 63r, 67v, 68v

24 Zeilen: 28r, 31r, 51v, 53r, 55r, 68r, 69rv, 70rv

29 Zeilen: 1v, 91r, 93v, 94r, 96r, 98v, 100r, 106r, 122v

30 Zeilen: 1r, 37rv, 92v, 93r, 94v, 95rv, 96v, 97v, 98r, 99r, 101r, 106v, 117rv, 118v, 119rv, 120v, 121r

31 Zeilen: 97r, 100v, 102rv, 103rv, 104v, 107v, 109v, 118r, 120r

32 Zeilen: 101v, 104r, 105v, 107r, 109r, 110r, 114rv, 116v

33 Zeilen: 105r, 108rv, 110v, 111r, 112rv, 115v

34 Zeilen: 111v, 113rv, 115r, 116r.

Die Zeilenzahl nimmt also gegen Ende der Handschrift zu.

Der Kodex ist von nur einer Hand mit dunkelbrauner Tinte in Buchkursive geschrieben. Die alte Bibliothekssignatur D.XIIII• ist von einer Hand des 15. Jahrhunderts in roter Tinte auf dem oberen Rand von Seite 1r vermerkt. Im Vorderdeckel steht am oberen Rand von moderner Hand die Signatur Cent. VIII,2 und darunter in deutscher Schrift 123 Blatter enthaltend. Die Blătter 124 und 125 sind nicht beschrieben.

Die Überschriften sind rot. Die roten Lombarden am Beginn der einzelnen Perikopen sind mehr-, zumeist zweizeilig und haben zum Teil ausgesparte Ornamente. Mitunter findet sich mitten im Text am Seitenanfang eine die Zeile überragende Lombarde. Einzelne Oberlängen - vor allem vom $\boldsymbol{d}$ - sind des öfteren in der ersten Zeile verlängert und mit Schnörkeln versehen. Mit einem senkrechten roten Strich sind die Anfangsbuchstaben der Wörter, mit denen ein Satz bzw. ein Satzteil beginnt, vom Rubrikator durchgehend, aber unregelmäbig und nicht immer konsequent gekennzeichnet. Namen sind zumeist - vor allem (christ)us und ih(es)us - mittels eines waagerechten roten Strichs durch das ganze Wort hervorgehoben.

Der alte Koperteinband besteht aus einem Pergamentumschlag, der Rücken ist mit zwei dunkelbraunen Lederstücken verstärkt. Zwei Lederknöpfe auf diesen Lederstücken dienen zum Verschließen, die übergreifende Klappe am Rückendeckel ist abgeschnitten. Im Vorderdeckel ist von der Hand der Schwester Kunigund Niklasin ${ }^{21}$ der Handschrifteninhalt vermerkt: It(em) an de(m) puch stet zu(m) erste(n) die Ew(an)gelio / Vn(d) die . iiij passio Vn(d) etlich Ew(an)gelio / vo(n) etlich heilige(n). Darunter am unteren Rand steht ein alter Bibliothekseintrag: Das puch gehort in das clost(er) / zu sa(n)t kathr(ein) pr(ediger) or(den) $i(n)$ nur (mbergk). Auf Seite 123v am unteren Rand wird dieser Eintrag wiederholt: Das puch gehort in das clost(er) zu / sa(n)t kathr(ein) predig(er) ord(en) i(n) núr(mbergk). Die Handschrift befand sich laut altem Bibliothekskatalog schon vor a.1428 im Nürnberger Katharinenkloster der Dominikanerinnen ${ }^{24}$.

24 Vgl. Mittelalterliche Bibliothekskataloge Deutschlands und der Schweiz. Bd. III,3: Bistum Bamberg, bearbeitet von P. RUF, München 1939, S. 604: "D XIII. Item ein puch in eym conpert; das helt in im die ewangelio und die III passio und etlich heiligen ewangeli. Das puch ist vor der reformyrung hynnen gewest." Keine weiterfuhrenden Angaben bei S. KRÄMER (wie Anm. 22), S. 623. 


\section{2. ÜBERLIEFERUNGSZUSAMMENHANG}

Die Frankfurter Handschrift, wie alle andern eine Abschrift, repräsentiert einen Text, der sprachlich als spätmittelhochdeutsch zu bestimmen ist und die älteste Stufe überliefert. Die Bremer Handschrift steht ihr zeitlich und räumlich am nächsten. Über das Elsaß - repräsentiert durch die erste Nürnberger Handschrift - ist dieses Perikopenbuch nach Nürnberg gelangt - repräsentiert durch die zweite Nürnberger Handschrift. Die Verbindung zum Katharinenkloster in Nürnberg ${ }^{25}$ dürfte über das Dominikanerinnenkloster Schönensteinbach ${ }^{26}$ bestanden haben, wie denn auch später von dort her das Nürnberger Kloster a.1428 reformiert worden ist $t^{2}$. Eine unmittelbare Abhängigkeit im Sinne von Vorlage und Abschrift besteht zwischen den vier Handschriften nicht. Am weitesten ab steht der letzte und jüngste Kodex, von dem allerdings nicht feststeht, daß er im Katharinenkloster geschrieben worden ist $^{28}$. Eine genauere stemmatische Fixierung der Textzeugen ist bei der hier vorliegenden Überlieferungslage nicht möglich. Es ist zudem mit mehreren nicht bezeugten Zwischenstufen, mit Einwirkung der Vulgata und mit Kontaminationen mit anderen Übersetzungen zu rechnen. Der Einfluß der Leben-Jhesu-Überlieferung ist dabei die auffalligste Abweichung von einer ungestörten Texttradierung ${ }^{29}$. Auch im Perikopenbestand gibt es trotz eines festen Kernbestandes vielfältige Abweichungen.

$25 \mathrm{Vgl}$. WALTER FrIES, Kirche und Kloster zu St. Katharina in Nümberg, MVGN 25 (1924), S. 1-143; besonders S. 14-26.

26 Dazu vgl. JOH[ANNES] vON SCHLUMBERgER (Hrsg.), Seraphin Dietler's Chronik des Klosters Schönensteinbach, Gebweiler 1897; M. BARTH, Handbuch der elsässischen Kirchen im Mittelalter, Straßburg 1963, Sp. 1255-1262.

27 Vgl. JоHANNES KIST, Klosterreform im spätmittelalterlichen Nürnberg, ZBKG 32 (1963), S. $34 f$.

28 Zum Skriptorium des Klosters im 14. und der ersten Hālfte des 15. Jahrhunderts vgl. K. SCHNEIDER / H. ZIRNBAUER (wie Anm. 19), S. XII.

29 Dazu im Exkurs S. LII-LV. 